\title{
Residue Number System Assisted Fast Frequency-Hopped Synchronous Ultra-Wideband Spread-Spectrum Multiple-Access: A Design Alternative to Impulse Radio
}

\author{
Lie-Liang Yang, Senior Member, IEEE and Lajos Hanzo, Senior Member, IEEE
}

\begin{abstract}
Ultra-wideband (UWB) systems having a bandwidth on the order of gigahertz have recently received wide attention both in the U.S. and in Europe. The family of UWB systems may communicate either by generating ultra-wideband signals or with the aid of innovatively combining conventional narrowband, wideband, or broadband signals. At the time of writing, UWB systems have only been implemented using ultra-wideband signals, such as those known from impulse radio systems. Hence, in this paper, UWB systems using narrowband signals are explored as a design alternative, which are based on the well-known family of frequency-hopping (FH) spread-spectrum multiple-access techniques. In the proposed UWB system, FH is implemented using multistage frequency-hpping multiple access (MS/UWB FHMA). We highlight the principles of the synchronous MS/UWB FHMA communication system, investigate the associated spectrum assignment, and the residue number system (RNS) based FH strategy. Detection of the received signal can be achieved with the aid of existing fast FH signal detection schemes. Our study shows that the RNS assisted FH strategy is capable of efficiently dividing the huge number of users supported by the synchronous MS/UWB FHMA system into a number of reduced-size user groups, where the multiuser interference only affects the users within the same group. Since the number of users in each group is only a small fraction of the total number of users supported by the synchronous MS/UWB FHMA system, advanced multiuser detection algorithms can be employed for achieving near-single-user performance at an acceptable complexity. Our results show that MS/UWB FHMA is capable of supporting an extremely high number of users, while employing relatively simple receivers.
\end{abstract}

Index Terms - Frequency-hopping (FH), multiuser detection (MUD), residue number system (RNS), spread-spectrum multiple access (SSMA), ultra-wide bandwidth radio.

\section{INTRODUCTION}

I T IS WIDELY recognized that in a data communications network the call blocking probability [1] decreases, when the number of channels each user is capable of accessing increases. Naturally, providing an increased number of traffic channels requires more bandwidth. The last decade has witnessed a paradigm shift from narrowband wireless systems to

Manuscript received November 22, 2001. This work was supported in part by the European Union under the Information Society Technologies (IST) Project IST 1999-12070 TRUST. This paper was presented in part at the International Conference on Communications, New York, NY, April 28-May 2, 2002.

The authors are with the Department of Electronics and Computer Science, University of Southampton, SO17 1BJ, U.K. (e-mail: 1ly@ecs.soton.ac.uk; 1h@ecs.soton.ac.uk).

Digital Object Identifier 10.1109/JSAC.2002.805059 broadband wireless systems [2], [3]. This trend continued with the recent emergence of the family of ultra-wideband (UWB) systems [4], which have received considerable attention both in North America [4]-[9] and in Europe.

UWB systems studied in the literature [4]-[8], [10]-[13] have been based on ultra-wideband signals using narrow timedomain impulses transmitted with the aid of time-hopping spread-spectrum techniques or position modulation. The UWB systems having the above characteristics are often referred to as impulse radio (IR) systems or time domain radio systems. However, UWB systems can also be implemented with the aid of conventional narrowband, wideband, or broadband signals. The typical advantages of using conventional signaling modes instead of UWB signaling modes when implementing UWB systems include but are not limited to the following features: 1) numerous well-understood and spectral-efficient techniques originally developed for conventional signaling modes may be employed by the proposed UWB system for achieving high communications efficiency; and 2) following this rationale, future UWB systems may become capable of using elements of the existing standards.

In this paper, an UWB system using narrowband signals is proposed. The proposed UWB system is capable of supporting a high number of users, while also allowing each user to access an ultra-wide bandwidth. The philosophy of the UWB multiple access scheme advocated is based on multistage frequency-hopping (FH) invoked in the context of noncoherent $M$-ary frequency shift keying (MFSK). Hence, we refer to the proposed scheme as multistage ultra-wideband frequency-hopping multiple-access and use the abbreviation of MS/UWB FHMA. In this paper, we highlight the principles of synchronous MS/UWB FHMA with particular emphasis on the associated spectrum assignment and FH strategy. More specifically, a residue number system (RNS) [14]-[17] assisted fast frequency-hopping (FFH) scheme is proposed for supporting the operation of the synchronous MS/UWB FHMA arrangement. The objective of using RNS based FFH in the proposed synchronous MS/UWB FHMA scheme is to ensure that the high number of users supported can be divided into a number of reduced-size user groups. The RNS based FFH principle guarantees that each MS/UWB FHMA user becomes capable of accessing an ultra-wide bandwidth, while ensuring that multiuser interference (MUI) is only imposed on users belonging to the same reduced-size user group. In our further discourse, we will show that the number of users 
in each group is significantly lower than the total number of users supported by the synchronous MS/UWB FHMA system. Hence, the receiver can afford invoking advanced multiuser detection (MUD) algorithms, in order to attain a single-user like performance. By contrast, without the above mentioned grouping the number of users would become excessive for supporting the employment of MUD. The bit-error rate (BER) performance of synchronous MS/UWB FHMA systems is investigated, when invoking existing MUD algorithms for communicating over MUI-contaminated environments. We assume that the active users' MS/UWB FHMA signals are transmitted synchronously, because this is usually the case for the down link (base-to-mobile) of a cellular system. We study the downlink performance, since it is expected to become the bottleneck in the emerging wireless Internet, which is likely to convey more file down-loading type traffic than uplink traffic. Another reason of using the synchronous assumption is that the transmitted narrowband signals in MS/UWB FHMA systems usually only experience flat-fading and in this scenario accurate synchronization can be readily achieved. We will demonstrate that synchronous MS/UWB FHMA constitutes a high-efficiency multiple access scheme capable of supporting an extremely high number of users with the aid of relatively simple receivers, which have been previously proposed in the literature [18]-[20].

In addition to the advantages mentioned above, the multistage FH technique advocated provides further unique advantages in the context of the system proposed, especially where low-power communication and high transmission robustness are key requirements. It is widely recognized that the spectrum of a FH-based system is reminiscent of that of a narrowband system during each hop. Detection has to be performed during each hop, which is significantly less demanding than demodulating during each chip interval, which would be necessitated both in direct-sequence code-division multiple access (DS-CDMA) and in time-division multiple access (TDMA). This potentially results in a low power consumption. Furthermore, since the hop-rate of FH systems is typically lower than the chip rate of DS systems, more time is available for synchronization and, hence, the synchronization requirements of FH systems are less stringent, than those of DS systems. Additionally, FH multiple-access (FHMA) systems are in general less susceptible to the near-far problem than DS systems [21] and the conventional single-user detector (SUD) of FHMA systems exhibits a high near-far resistance in the synchronous case [21]. Furthermore, in contrast to conventional UWB IR [4], [8], which has a spectrum overlapping with the currently used radio frequency bands and, hence, inevitably inflicts a certain amount of intersystem interference, the frequency band occupied by $\mathrm{FH}$ systems does not have to be contiguous. Hence, the frequency bands affected by severe fading or those bands that are used by another system can be avoided.

The remainder of this paper is organized as follows. In Section II, we describe the proposed MS/UWB FHMA system along with its transmitter and spectrum assignment philosophy. Then, in Section III, we consider the receiver of the MS/UWB FHMA system and discuss the detection of MS/UWB FHMA signals in the context of both conventional single-user and advanced MUD. In Section IV, the BER performance of the proposed MS/UWB FHMA system using different detection schemes is investigated using simulations. Finally, we provide our conclusions in Section $\mathrm{V}$ and indicate potential further research topics.

\section{SYSTEM DESCRIPTION}

\section{A. Transmitter}

The operation of the transmitter associated with the MS/UWB FHMA system studied may be understood by referring to Fig. 1. During a signaling interval of $T_{s}$ duration, $b$ message bits of the $k$ th user having a bit rate of $R_{b}$ are loaded into a $b$-bit buffer. We denote this $b$-bit symbol as $X_{k}^{(m)}$, where $X_{k}^{(m)} \in\left[0, M=2^{b}\right)$. Hence, the $b$-bit output symbol rate is $R_{s}=R_{b} / b$. Let $R_{h}=1 / T_{h}$ be the FH rate, where $T_{h}$ represents the duration of the $\mathrm{FH}$ tones or chips. The ratio of $L=T_{s} / T_{h}$ determines, whether slow FH (SFH)-where $L<1$-or fast FH (FFH) - where $L \geq 1$-is invoked. Specifically, if $L<1$, several $M=2^{b}$-ary symbols are transmitted in a FH-interval of $T_{h}$ duration, activating one out of $M$ legitimate frequencies of an MFSK scheme. By contrast, if $L \geq 1$ and integer, an $M=2^{b}$-ary symbol activates a particular MFSK tone only for the duration of $T_{h}=T_{s} / L$ seconds and then hops to a further $(L-1)$ other legitimate frequencies of the MFSK scheme used.

In our proposed system, we assume that $L=T_{s} / T_{h}$ is an integer larger than one, i.e., that FFH is employed [18]. In order to distinguish between different users, each user is assigned a user-signature $\mathrm{FH}$ pattern also referred to as an address code [18]-[20]. Let the address code of the $k$ th user, $1 \leq k \leq K$, be expressed as $\mathbf{a}_{k}=\left[a_{k}(0), a_{k}(1), \ldots, a_{k}(L-1)\right]$, where $a_{k}(l) \in G F(M), l=0,1, \ldots, L-1$, and $G F(M)$ denotes a Galois field over $M=2^{b}$. Then, the transmitted symbol $X_{k}^{(M)}$ is signaled by invoking the $k$ th user's address code, which can be expressed as

$$
\mathbf{Y}_{k}=\left[y_{k}(0), y_{k}(1), \ldots, y_{k}(L-1)\right]=X_{k}^{(m)} \cdot \mathbf{1} \oplus \mathbf{a}_{k}
$$

where 1 is a unit vector of length $L, y_{k}(l)$ for $1 \leq l<L$ are elements of $G F(M)$, and $\oplus$ denotes modulo- $M$ or modulo- $2^{b}$ addition, as seen in Fig. 1. Obeying the user signature, the components of $\mathbf{Y}_{k}$ are passed serially to an MFSK modulator, where they are converted to the MFSK tones $\mathbf{F}_{m}^{(k)}=\left[f_{d, 0}^{(k)}, f_{d, 1}^{(k)}, \ldots, f_{d, L-1}^{(k)}\right]$ referred to as chips of duration $T_{h}$. Following MFSK modulation FH is activated obeying the user-specific FH pattern derived using the multistage FH scheme to be detailed in the next subsection during our forthcoming discourse. Finally, the modulated signal is transmitted using the RF mixing stage. During the time interval $t \in\left[i T_{s},(i+1) T_{s}\right]$, the $k$ th user's transmitted signal can be expressed as

$$
\begin{aligned}
s_{k}^{(i)}(t)= & \sum_{l=0}^{L-1} \sqrt{2 P} p_{T_{h}}\left(t-i T_{s}-l T_{h}\right) \\
& \cdot \cos \left(2 \pi\left[\sum_{s=1}^{S} f_{s, i_{s}, l}^{(k)}+f_{d, l}^{(k)}\right] t+\psi_{i, l}^{(k)}\right)
\end{aligned}
$$

where $P$ is the transmitted power, $p_{T_{h}}(t)$ is the pulse-shaped waveform associated with a chip defined over the interval 


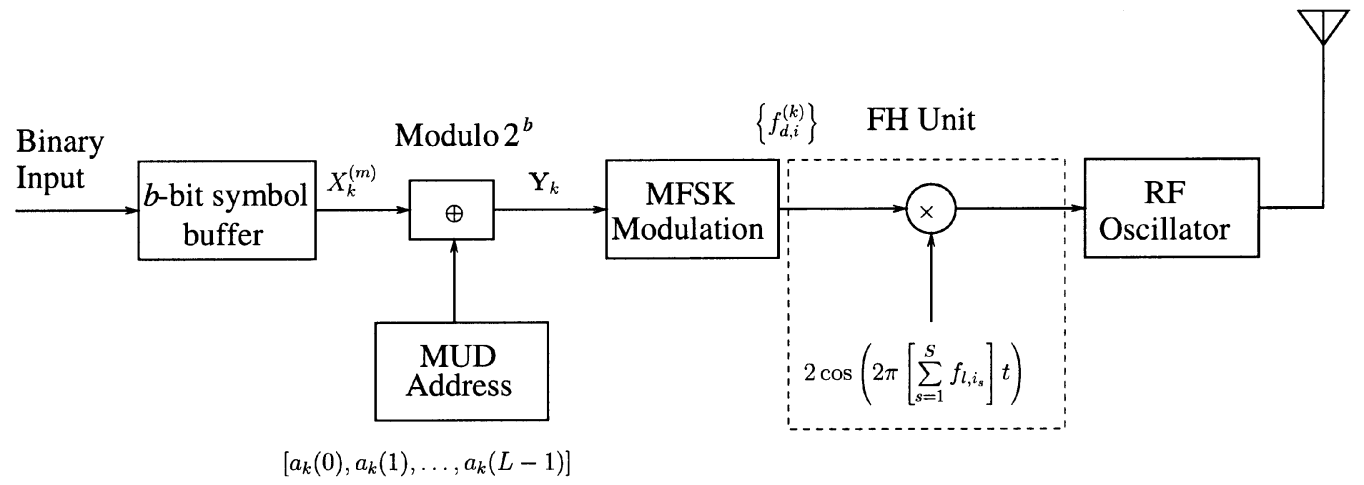

(a)

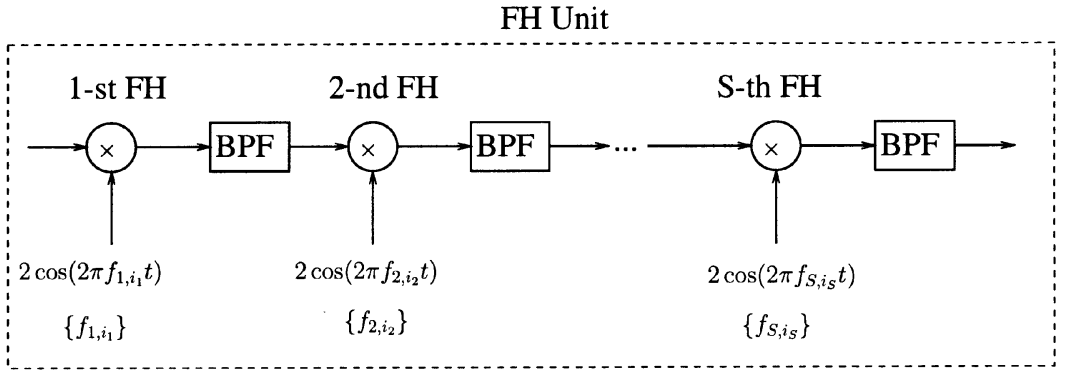

(b)

Fig. 1. Transmitter block diagram of the MS/UWB FHMA system using MFSK modulation.

$\left[0, T_{h}\right), S$ represents the number of FH stages seen in Fig. 1(b) and $f_{s, i_{s}, l}^{(k)}$ represents the base frequency corresponding to the $i$ th transmitted symbol in the $s$ th FH stage, $s=1,2, \ldots, S$ during the $l$ th chip interval. Finally, $\psi_{i, l}^{(k)}$ represents the phase introduced by the MFSK modulation and FH procedures.

The concept of multistage FH can be augmented with the aid of Figs. 1(b) and 2. As an example, we characterize a three-stage FHMA system associated with 16-ary MFSK modulation, which will be further detailed during our forthcoming discourse. In the MS/UWB FHMA system, the total bandwidth of $W_{s s}$ is first uniformly divided into $m_{S}$ number of frequency bands, each having a bandwidth of $W_{F H}^{S}=W_{s s} / m_{S}$-which is referred to as the Stage- $S$ subband — and each subband is correspondingly assigned a frequency from the Stage- $S$ frequency set expressed as $\mathbf{F}_{S}=\left[f_{S, 0}, f_{S, 1}, \ldots, f_{S, m_{S}-1}\right]$. In the example of Fig. 2 this corresponds to Stage-3 at the top. Then, the Stage- $S$ subband is further divided, again, uniformly into $m_{S-1}$ number of frequency bands, where each band has a bandwidth of $W_{F H}^{S-1}=W_{F H}^{S} / m_{S-1}$. We refer to $W_{F H}^{S-1}$ as the Stage- $(S-1)$ subband, which is referred to as Stage-2 in the example of Fig. 2. Following the procedure used at Stage- $S$, each of the Stage- $(S-1)$ subbands is assigned a frequency from the Stage- $(S-1)$ frequency set expressed as $\mathbf{F}_{(S-1)}=\left[f_{(S-1), 0}, f_{(S-1), 1}, \ldots, f_{(S-1), m_{(S-1)}-1}\right]$. According to the above rules, finally, the Stage-2 subband is uniformly divided into $m_{1}$ number of frequency bands, each having a bandwidth of $W_{F H}^{1}=W_{F H}^{2} / m_{1}$, which are referred to as the Stage- 1 subbands. At the same time, each of the Stage-1 subbands is assigned a frequency from the Stage-1 frequency set of $\mathbf{F}_{1}=\left[f_{1,0}, f_{1,1} \ldots, f_{1, m_{1}-1}\right]$.

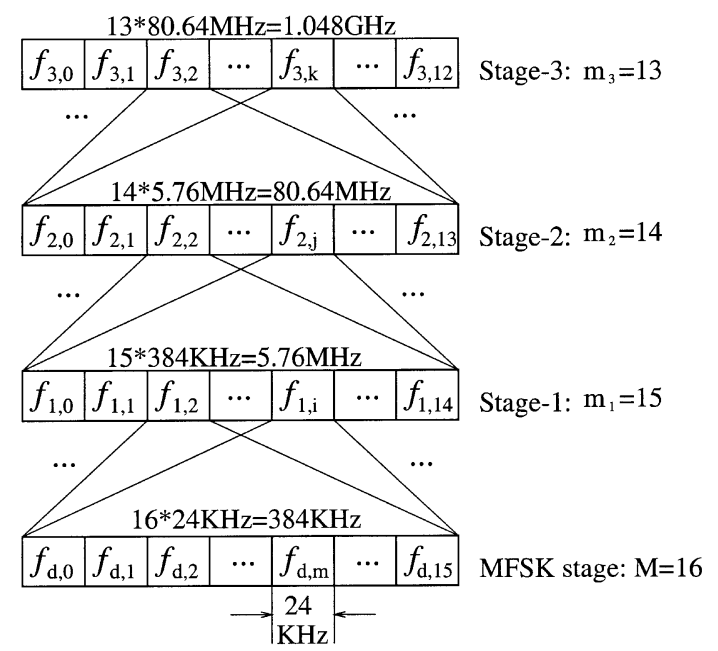

Fig. 2. Example of the spectrum allocation of a three-stage $(L=3)$ FH SSMA system using 16-ary MFSK modulation, where $m_{1}=15, m_{2}=14, m_{3}=13$, and $R_{b}=32 \mathrm{~Kb} / \mathrm{s}$.

Based on the above bandwidth assignment, the total number of FH bands is $M_{t}=\prod_{s=1}^{S} m_{s}$. As shown in Fig. 2, each FH band consists of $M$ tones associated with MFSK modulation. We referred to this processing stage as the MFSK stage. Since the FH rate is $R_{h}$, the spacing between two adjacent MFSK tones should be no less than $R_{h}$, which is an often used system constraint in FFH [18]. Consequently, the total system bandwidth $W_{s s}$ and the FH rate $R_{h}$ must obey the relationship of $W_{s s} \geq \prod_{s=1}^{S} m_{s} \times M R_{h}$. Since we have $R_{h}=L R_{s}=L R_{b} / b$, where $R_{s}$ and $R_{b}$ are the symbol rate and bit rate, respectively, 
the relationship of $W_{s s} \geq \prod_{s=1}^{S} m_{s} \times M L R_{b} / b$ holds. We assume that the FH rate at each FH stage is $R_{H}=1 / T_{H}$, i.e., the frequencies at a given FH stage $s, s=1,2, \ldots, S$ hop $\omega=T_{s} / T_{H}$ times per $M=2^{b}$-ary symbol. For example, $\omega=1$ would imply that the FH rate $R_{H}$ at each FH stage is the same as the symbol rate $R_{s}$, i.e., $R_{H}=R_{S}$. Furthermore, we assume that the relative FH delay between two adjacent FH stages is $T_{H} / S$, i.e., that the FH at the $(s+1)$ th stage happens $T_{H} / S$ seconds earlier or later than that at the $s$ th stage. Consequently, the actual number of hops (chips) per symbol, $L$, the number of hops, $\omega$, at each FH stage, as well as the number of FH stages, $S$, obey the relationship of $L=T_{s} /\left(T_{H} / S\right)=\omega S$.

Based on the above spectrum assignment strategy and assumptions, multistage FH can be implemented as follows. After the MFSK modulation stage of Fig. 1, each MFSK tone is frequency-hopped using $S$ number of possible frequencies from the $S$ frequency sets, one from each set, according to the FH strategy to be outlined in the next subsection. The FH frequency to be activated during each of the $L$ chips is determined by the sum of the frequencies activated at the $S$ number of FH stages, i.e., $\sum_{s=1}^{S} f_{s, i_{s}, l}$ as shown in the Fig. 1.

\section{B. RNS-Based FFH}

It is widely recognized that in FHMA communications the FH patterns must be assigned such that [22]: 1) there is no ambiguity concerning the identity of the user and the information it transmits; 2) the probability of a hit or collision between the frequencies activated by any two FH patterns is as low as possible, which implies that the derived user must interfere as small a fraction of time as possible with the other users; 3) all frequencies in the FH band are activated with the same probability; and 4) it is also desirable for the transmitter to hop to a frequency far from the previous one, i.e., consecutively activated MFSK tones of the FH patterns must be spaced sufficiently far apart, so that the channel's characteristics associated with consecutive hops become independent. If this requirement is met, the transmitter exhibits improved resistance against various sources of interference, such as partial-band interference, multitone interference as well as intersymbol interference. If the fading characteristics of consecutively activated MFSK tones can be considered uncorrelated, we can mitigate the effect of fading by using frequency diversity techniques. In the literature various methods of constructing $\mathrm{FH}$ patterns have been investigated [22]-[25] in the context of single-stage FH schemes. These methods of constructing FH patterns are based on generalized maximum-length linear-feedback shift register sequences ( $m$ sequences) [22], on invoking Reed-Solomon (RS) codes [22], or on the number-theoretic concept of congruence equations [24]. Furthermore, the set of FH sequences exhibiting a high linear span has been studied in [23], while the family of the "one-coincidence FH sequences" guaranteeing that any adjacent MFSK tones in any FH pattern are spaced by a specific minimum amount have been investigated in [25]. However, these $\mathrm{FH}$ sequences are only suitable for conventional single-stage FH systems. In this section, a FH scheme based on the RNS suitable for the proposed synchronous multistage FHMA system is contrived. Our investigations show that when synchronous transmissions are assumed, the FH patterns derived from this $\mathrm{FH}$ scheme are capable of satisfying all the desirable FH pattern design criteria. However, the design of FH patterns for employment in asynchronous MS/UWB FHMA systems constitutes an open problem, requiring further investigations. Let us now describe the FH scheme proposed for the family of synchronous MS/UWB FHMA systems by first giving a brief introduction to the basic philosophy of RNSs.

A RNS [26] is defined by the choice of $S$ positive integers $m_{1}, m_{2}, \ldots, m_{S}$ referred to as moduli. If all the moduli are pairwise relative primes, any integer $\mathbf{A}_{k}$-which is used to describe the $k$ th user's FH address, can be uniquely and unambiguously represented by the residue sequence $\mathbf{A}_{k} \Leftrightarrow\left(A_{k 1}, A_{k 2}, \ldots, A_{k S}\right)$ in the range of $0 \leq \mathbf{A}_{k}<M_{S}$, where $A_{k s}=\mathbf{A}_{k}\left(\bmod m_{s}\right)$ represents the residue digits of $\mathbf{A}_{k}$ upon division by $m_{s}$ and $M_{S}=\prod_{s=1}^{S} m_{s}$ is the dynamic range of the RNS. By contrast, the notation $\mathbf{a}_{k}$ will be used in the context of MUD to be introduced in Section III, which will be referred to as the $k$ th user's MUD address. According to the Chinese remainder theorem (CRT) [27], for any given $S$-tuple $\left(A_{k 1}, A_{k 2}, \ldots, A_{k S}\right)$, where $0 \leq A_{k s}<m_{s}$, there exists one and only one integer $\mathbf{A}_{k}$ such that $0 \leq \mathbf{A}_{k}<M_{S}$ and $A_{k s}=\mathbf{A}_{k}\left(\bmod m_{s}\right)$, which allows us to uniquely recover the integer value of $\mathbf{A}_{k}$ from the received residue digits. Again, $\mathbf{A}_{k}$ represents the $\mathrm{FH}$ address of user $k$.

Assuming that the integers $\mathbf{A}_{\mathbf{1}}$ and $\mathbf{A}_{\mathbf{2}}$ have RNS representations of $\mathbf{A}_{\mathbf{1}} \Leftrightarrow\left(A_{11}, A_{12}, \ldots, A_{1 S}\right)$ and $\mathbf{A}_{\mathbf{2}} \Leftrightarrow\left(A_{21}, A_{22}, \ldots, A_{2 S}\right)$, respectively, then $\mathbf{A}_{\mathbf{1}} \bullet \mathbf{A}_{\mathbf{2}}$, where $\bullet$ denotes addition, subtraction or multiplication, yields another unique residue sequence $\mathbf{A}_{\mathbf{3}}$. The arithmetic operations over the RNS can be carried out on a residue-by-residue basis, which can be expressed as [26]

$$
\mathbf{A}_{\mathbf{3}}=\mathbf{\mathbf { A } _ { \mathbf { 1 } }} \bullet \mathbf{\mathbf { A } _ { 2 }}\left(\bmod M_{S}\right) \Leftrightarrow\left[\left(A_{1 s} \bullet A_{2 s}\right)\left(\bmod m_{s}\right)\right]_{s=1}^{S}
$$

where on the left of (3) the operation $\bullet$ represents modulo- $M_{s}$ addition, subtraction, or multiplication of two integers and at the right of (3) • represents the same operations performed on the basis of the appropriate residue digits $A_{1 s}$ and $A_{2 s}$, with respect to their corresponding modulus $m_{s}$.

Single-User FH: The basic principle of the RNS-based multistage FH scheme is that the frequencies at the $S$ number of stages are activated according to the $S$ number of residue digits of a corresponding residue sequence in the RNS. Specifically, let $\mathbf{A}_{k}^{i} \Leftrightarrow\left(A_{k 1}^{i}, A_{k 2}^{i}, \ldots, A_{k S}^{i}\right)$ be the residue sequence representation of integer $\mathbf{A}_{k}^{i}$ in terms of a RNS using moduli $m_{i}, m_{2}, \ldots, m_{S}$, where the superscript $i$ represents the time index. Then the FH frequency at instant $i$ is determined by the sum of $f_{1, A_{k 1}^{i}}$ from the Stage-1 frequency set $\mathbf{F}_{1}, f_{2, A_{k 2}^{i}}$ from the Stage-2 frequency set $\mathbf{F}_{2}$, an so on until $f_{S, A_{k S}^{i}}$ from the Stage- $S$ frequency set $\mathbf{F}_{S}$, i.e., by $\sum_{s=1}^{S} f_{s, A_{k s}^{i}}$. At the time index $(i+1), \mathbf{A}_{k}^{i}$ is increased by one and, hence, it is represented in the RNS domain as $\mathbf{A}_{k}^{(i+1)}=\mathbf{A}_{k}^{i}+1 \Leftrightarrow\left(A_{k i}^{(i+1)}, A_{k 2}^{(i+1)}, \ldots, A_{k S}^{(i+1)}\right)$. Similarly, the FH frequency at instant $(i+1)$ is determined by the sum of $f_{1, A_{k 1}^{(i+1)}}$ from the Stage-1 frequency set $\mathbf{F}_{1}, f_{2, A_{k 2}^{(i+1)}}$ from the 
Stage-2 frequency set $\mathbf{F}_{2}$, etc. until $f_{S, A_{k S}^{(i+1)}}$ from the Stage- $S$ frequency set $\mathbf{F}_{S}$, yielding $\sum_{s=1}^{S} f_{s, A_{k s}^{(i+1)}}$. The above procedure is continued until the end of transmission of user $k$. Since the dynamic range of the above RNS is $\left[0, M_{s}=\prod_{s=1}^{S} m_{s}\right)$, the period of the FH patterns is $M_{S}$.

Multiuser FHMA: Let us now discuss the RNS-based MS/UWB FHMA scheme. Let $m_{1}, m_{2}, \ldots, m_{S}$ be the pairwise relative primes representing the number of frequencies in the $S$ number of frequency sets, namely in $\mathbf{F}_{1}, \mathbf{F}_{2}, \ldots, \mathbf{F}_{S}$, with respect to the $S \mathrm{FH}$ stages of Fig. 2, respectively. Let $\mathbf{A}_{k} \Leftrightarrow\left(A_{k 1}, A_{k 2}, \ldots, A_{k S}\right)$, where $0 \leq \mathbf{A}_{k}<M_{S}$ and $\mathbf{a}_{k}=\left[a_{k}(0), a_{k}(1), \ldots, a_{k}(L-1)\right]$ be the FH address and MUD address of user $k$, respectively. Furthermore, let $\mathbf{C} \Leftrightarrow\left(C_{1}, C_{2}, \ldots, C_{S}\right)$ represent a reference register in the RNS domain, which provides reference to each user, in order to implement synchronous transmission. $\mathbf{C}$ is increased by one, whenever $S$ hops happened. According to (3), this can be implemented by increasing each element of $\left(C_{1}, C_{2}, \ldots, C_{S}\right)$ by one with respect to their corresponding modulus. Note that although the maximum integer represented by the RNS-based registers $\left(C_{1}, C_{2}, \ldots, C_{S}\right)$ is $M_{S}-1=\prod_{s=1}^{S} m_{s}-1$, this will not prevent us from using it as a synchronous transmission reference. Indeed, this property can substantially simplify the implementation of synchronous transmissions, as we will show below. According to the above assumptions, when the $k$ th user is attempting to access the network and to transmit the information symbol $X_{k}^{(m)}$, the operation of the multistage FHMA scheme can be described with the aid of a few formally defined, but heuristically contrived steps, which will be introduced below and explicitly augmented with the aid of a numerical example during our further discourse. We note that the reader may find it beneficial to consult the numerical example following our general algorithm cast in terms of the following steps.

Step 1) According to (1), $X_{k}^{(m)}$ is signaled with the aid of the $k$ th user's MUD address with respect to the chips $0,1, \ldots, L-1$, where $L=\omega S$. The $M$-ary symbols are expressed as $\mathbf{Y}_{k}=\left[y_{k}(0), y_{k}(1), \ldots, y_{k}(L-1)\right]=$ $X_{k}^{(m)} \cdot \mathbf{1} \bigoplus \mathbf{a}_{k}$, which are signaled using the $L$ number of chips each hosting one of the $M$ possible signaling tones.

Step 2) Based on the $k$ th user's FH address $\mathbf{A}_{k}$ and on the reference register's instantaneous value of $\mathbf{C}$, we derive the residue sequence $\mathbf{I}_{k}$ as follows:

$\mathbf{I}_{k} \Leftrightarrow\left(i_{1}, i_{2}, \ldots, i_{S}\right)=\left[\left(A_{k s} \oplus C_{s}\right)\left(\bmod m_{s}\right)\right]_{s=1}^{S}$

according to (3).

Step 3) For the chip $t S \leq l<(t+1) S, t \leq \omega-1$, the transmitted frequency is constituted by the sum of the frequencies $f_{1, i_{1}+t}$ from the Stage-1 frequency set $\mathbf{F}_{1}, f_{2, i_{2}+t}$ from the Stage-2 frequency set $\mathbf{F}_{2}$, etc., up to $f_{S-(l-t S)-1, i_{S-(l-t S)-1}+t}$ from the Stage- $(S-(l-t S)-1)$ frequency set $\mathbf{F}_{S-(l-t S)-1}$ and the frequencies $f_{S-(l-t S), i_{S-(l-t S)}+t+1}$ from the Stage- $(S-(l-t S))$ frequency set $\mathbf{F}_{S-(l-t S)}$ up to $f_{S, i_{S}+t+1}$ from the Stage- $S$ frequency set $\mathbf{F}_{S}$, as well as the frequency $f_{d, l}^{(k)}$ corresponding to the MFSK tone activated according to $y_{k}(l)$. The frequency given by the corresponding sum can be expressed as

$$
f_{k, l}=\sum_{j=1}^{S-(l-t S)-1} f_{j, i_{j}+t}+\sum_{j=S-(l-t S)}^{S} f_{j, i_{j}+t+1}+f_{d, l}^{(k)} .
$$

Step 4) The remaining MFSK symbols are transmitted by repeating the above three steps.

The philosophy of the above FH procedure may be augmented with the aid of an example. In Table I, we characterize a synchronous multistage FHMA scheme assuming $S=L$ and $\omega=1$, where the first and second user's FH addresses are $17 \Leftrightarrow(4,3,2)$ and $89 \Leftrightarrow(11,5,14)$, respectively, while the initial value of the RNS-based reference register is $(10,10,10)$, all three quantities given in terms of the moduli $m_{3}=13, m_{2}=14, m_{1}=15$. Furthermore, in Table I, we assumed that the first user entered the system at $t=0$, while the second user entered the system at $t=3 T_{s}$. As shown in Table I, when the first user enters the system at $t=0$, the residue sequence $\mathbf{I}_{1} \Leftrightarrow(1,13,12)$ is generated according to (4). Then, according to Step 3 the FH frequencies activated during the chips 0,1 , and 2 of the first $M$-ary symbol are $f_{3,2}+f_{2,13}+f_{1,12}, f_{3,2}+f_{2,0}+f_{1,12}$, and $f_{3,2}+f_{2,0}+f_{1,13}$, respectively, where $f_{3, i}, f_{2, j}, f_{1, k}$ belong to the Stage-3, Stage-2, and Stage-1 frequency sets $\mathbf{F}_{3}, \mathbf{F}_{2}$, and $\mathbf{F}_{1}$, respectively. Let $f_{d, 0}^{(1)}, f_{d, 1}^{(1)}$ and $f_{d, 2}^{(1)}$ be the MFSK tones corresponding to the chips 0,1 , and 2 of the first symbol, which are activated according to $\mathbf{Y}_{1}=\left[y_{1}(0), y_{1}(1), y_{1}(2)\right]$ at the MFSK stage. According to (5) the actually transmitted frequency during a given chip is constituted by the sum of the FH frequencies at the $S$ FH stages plus the frequency of the MFSK stage. Hence, the actually transmitted frequencies during the chips 0,1 , and 2 of the first $M$-ary symbol are $f_{3,2}+f_{2,13}+f_{1,12}+f_{d, 0}^{(1)}, f_{3,2}+f_{2,0}+f_{1,12}+f_{d, 1}^{(1)}$, and $f_{3,2}+f_{2,0}+f_{1,13}+f_{d, 2}^{(1)}$. Following the last chip of the first $M$-ary symbol, the common RNS-based reference register's value is increased by one. The remaining symbols of the first user can be transmitted similarly as described above. As shown in Table I, when the second user enters the system at $t=3 T_{s}$, the residue sequence $\mathbf{I}_{2} \Leftrightarrow(11,4,12)$ is generated according to (4) as the sum of its FH address $89 \Leftrightarrow(11,5,14)$ and the instantaneous value $\mathbf{C}=(0,13,13)$ of the RNS-based reference register at $t=3 T_{s}$. As seen in Table $\mathrm{I}$, the elements of the residue sequence $\mathbf{I}_{2} \Leftrightarrow(11,4,12)$ correspond to the frequencies $f_{3,11}, f_{2,4}, f_{1,12}$ of the $S=3 \mathrm{FH}$ stages of Fig. 2. The rest of the transmission procedure is similar to that of the first user. With the aid of Table I, we can see that each user employs a different composite frequency during each chip and the $M_{s}=\prod_{s=1}^{S} m_{s}$ number of $\mathrm{FH}$ frequencies of the total UWB FH bands are used with the same probability, since the above FH scheme generates a FH pattern having a period of $M_{s}$ hops. Finally, we can infer from Table I that user 1 and user 2 will never collide with each other. This is because the FH addresses of user 1 and user 2 are different, as well as because the composite frequencies of user 1 and user 2 in any chip are derived from their FH address after adding these FH addresses to the same instantaneous value of the common RNS-based reference register. 
TABLE I

EXAMPLE OF THE RNS-BASED MS/UWB FHMA SYSTEM, WHERE THE FH ADDRESSES OF THE FIRST AND SECOND USERS ARE 17 AND 89, ResPeCtiVELy, While the INITIAL VALUE OF THE RNS-BASED ReFERENCE REGISTER IS (10, 10, 10) With RESPECT TO THE MODULI $m_{3}=13, m_{2}=14, m_{1}=15$

\begin{tabular}{|c|c|c|c|c|c|c|c|c|c|c|}
\hline \multirow{2}{*}{$\begin{array}{l}\text { Trans- } \\
\text { mitted }\end{array}$} & \multirow[b]{2}{*}{ FH } & \multicolumn{3}{|c|}{ User $1(17 \Leftrightarrow(4,3,2))$} & \multicolumn{3}{|c|}{ User $2(89 \Leftrightarrow(11,5,14))$} & \multicolumn{3}{|c|}{ Calculator } \\
\hline & & $\left(m_{3}\right)$ & $\left(m_{2}\right)$ & $\left(m_{1}\right)$ & $\left(m_{3}\right)$ & $\left(m_{2}\right)$ & $\left(m_{1}\right)$ & $\left(m_{3}\right)$ & $\left(m_{2}\right)$ & $\left(m_{1}\right)$ \\
\hline \multirow[t]{2}{*}{ Symbols } & Timing & 13 & 14 & 15 & 13 & 14 & 15 & 13 & 14 & 15 \\
\hline & $t=0$ & $f_{3,1}$ & $f_{2,13}$ & $f_{1,12}$ & & & & 10 & 10 & 10 \\
\hline Symbol & $T_{h}$ & $f_{3,2}$ & $f_{2,13}$ & $f_{1,12}$ & & & & & & \\
\hline \multirow[t]{2}{*}{1} & $2 T_{h}$ & $f_{3,2}$ & $f_{2,0}$ & $f_{1,12}$ & & & & & & \\
\hline & $3 T_{h}=T_{s}$ & $f_{3,2}$ & $f_{2,0}$ & $f_{1,13}$ & & & & 11 & 11 & 11 \\
\hline Symbol & $4 T_{h}$ & $f_{3,3}$ & $f_{2,0}$ & $f_{1,13}$ & & & & & & \\
\hline \multirow[t]{2}{*}{2} & $5 T_{h}$ & $f_{3,3}$ & $f_{2,1}$ & $f_{1,13}$ & & & & & & \\
\hline & $6 T_{h}=2 T_{s}$ & $f_{3,3}$ & $f_{2,1}$ & $f_{1,14}$ & & & & 12 & 12 & 12 \\
\hline Symbol & $7 T_{h}$ & $f_{3,4}$ & $f_{2,1}$ & $f_{1,14}$ & & & & & & \\
\hline \multirow[t]{2}{*}{3} & $8 T_{h}$ & $f_{3,4}$ & $f_{2,2}$ & $f_{1,14}$ & & & & & & \\
\hline & $9 T_{h}=3 T_{s}$ & $f_{3,4}$ & $f_{2,2}$ & $f_{1,0}$ & $f_{3,11}$ & $f_{2,4}$ & $f_{1,12}$ & 0 & 13 & 13 \\
\hline \multirow{3}{*}{$\begin{array}{c}\text { Symbol } \\
4\end{array}$} & $10 T_{h}$ & $f_{3,5}$ & $f_{2,2}$ & $f_{1,0}$ & $f_{3,12}$ & $f_{2,4}$ & $f_{1,12}$ & & & \\
\hline & $11 T_{h}$ & $f_{3,5}$ & $f_{2,3}$ & $f_{1,0}$ & $f_{3,12}$ & $f_{2,5}$ & $f_{1,12}$ & & & \\
\hline & $12 T_{h}=4 T_{s}$ & $f_{3,5}$ & $f_{2,3}$ & $f_{1,1}$ & $f_{3,12}$ & $f_{2,5}$ & $f_{1,13}$ & 1 & 0 & 14 \\
\hline \multirow{3}{*}{$\begin{array}{c}\text { Symbol } \\
5\end{array}$} & $13 T_{h}$ & $f_{3,6}$ & $f_{2,3}$ & $f_{1,1}$ & $f_{3,0}$ & $f_{2,5}$ & $f_{1,13}$ & & & \\
\hline & $14 T_{h}$ & $f_{3,6}$ & $f_{2,4}$ & $f_{1,1}$ & $f_{3,0}$ & $f_{2,6}$ & $f_{1,13}$ & & & \\
\hline & $15 T_{h}=5 T_{s}$ & $f_{3,6}$ & $f_{2,4}$ & $f_{1,2}$ & $f_{3,0}$ & $f_{2,6}$ & $f_{1,14}$ & 2 & 1 & 0 \\
\hline \multirow{3}{*}{$\begin{array}{c}\text { Symbol } \\
6\end{array}$} & $16 T_{h}$ & $f_{3,7}$ & $f_{2,4}$ & $f_{1,2}$ & $f_{3,1}$ & $f_{2,6}$ & $f_{1,14}$ & & & \\
\hline & $17 T_{h}$ & $f_{3,7}$ & $f_{2,5}$ & $f_{1,2}$ & $f_{3,1}$ & $f_{2,7}$ & $f_{1,14}$ & & & \\
\hline & $18 T_{h}=6 T_{s}$ & $f_{3,7}$ & $f_{2,5}$ & $f_{1,3}$ & $f_{3,1}$ & $f_{3,7}$ & $f_{1,0}$ & 3 & 2 & 1 \\
\hline
\end{tabular}

According to the above principles, if the number of users satisfies $K \leq M_{s}, K$ out of the $M_{s}$ FH addresses, which are expressed in the form of residue sequences derived from the integers $0,1, \ldots, M_{s}-1$, can be uniquely assigned to the $K$ users. Again, based on this unique assignment and on the RNS based FHMA scheme described above, it can be readily shown that no collisions occur between any two users, since at any moment, the addition of these FH addresses to the same instantaneous value of the common RNS-based reference register will generate $K \leq M_{s}$ different integers in the range of $\left[0, M_{s}\right)$. Consequently, the hopping frequencies derived from the resultant $K$ integers represented as $K$ residue sequences are unique. Therefore, again, if the number of users obeys $K \leq M_{s}$, the probability of hits or collisions is zero.

However, the MS/UWB FHMA system is capable of supporting more than $K$ users, if efficient MUD techniques can be employed. More explicitly, the $S$-stage MS/UWB FHMA system using the RNS moduli $\left(m_{1}, m_{2}, \ldots, m_{s}\right)$ and MFSK modulation can support up to $K=M \times \prod_{s=1}^{S} m_{s}$ number of users by invoking MUD techniques [19], [20]. If the number of users, $K$, is higher than the number of unique FH addresses, $M_{S}$, i.e., $K>M_{S}$, then a given FH address can be assigned to a group of users and the users in this group are distinguished by their corresponding MUD address, $\mathbf{a}_{k}=\left[a_{k}(0), a_{k}(1), \ldots, a_{k}(L-1)\right], k=1,2, \ldots, K^{\prime}$. Therefore, the set of users sharing the same FH address are referred to as a MUD group. More explicitly, if $U<K / M_{s} \leq U+1 \leq M$, then a given $\mathrm{FH}$ address has to be assigned to more than one, namely to $U$ or $U+1$ users, which form a MUD group. In order to resolve the associated ambiguity, an additional set of $U$ or $(U+1)$ MUD addresses are assigned employing a unique mapping to each of the $U$ or the $(U+1)$ users in the same user group, in order to distinguish them from each other. Note that, the same MUD address can be assigned to several users belong to different groups, since the groups are differentiated with the aid of the unique FH addresses. Based on the above user signature assignment rule, if nonfading channels or frequency nonselective fading channels are encountered by each MFSK tone, then the multiuser detector of the synchronous MS/UWB FHMA system only has to differentiated among the users of the same group, i.e., the users that were assigned the same FH address. Consequently, the number of users detected by the multiuser receiver is about $K^{\prime}=K / M_{s}$, which is not higher than $M$ and significantly lower than $K$ representing the total number of users supported by the MS/UWB FHMA system. Therefore, the complexity of the multiuser receiver employed in the MS/UWB FHMA system can be substantially decreased in comparison to that of the conventional MUD concept, where all system users are detected simultaneously.

Furthermore, by referring to (5) and with the aid of Table I, we observe that the MFSK tones are activated at a rate of $R_{h}$. In other words, during each chip, one of $M$ legitimate MFSK 


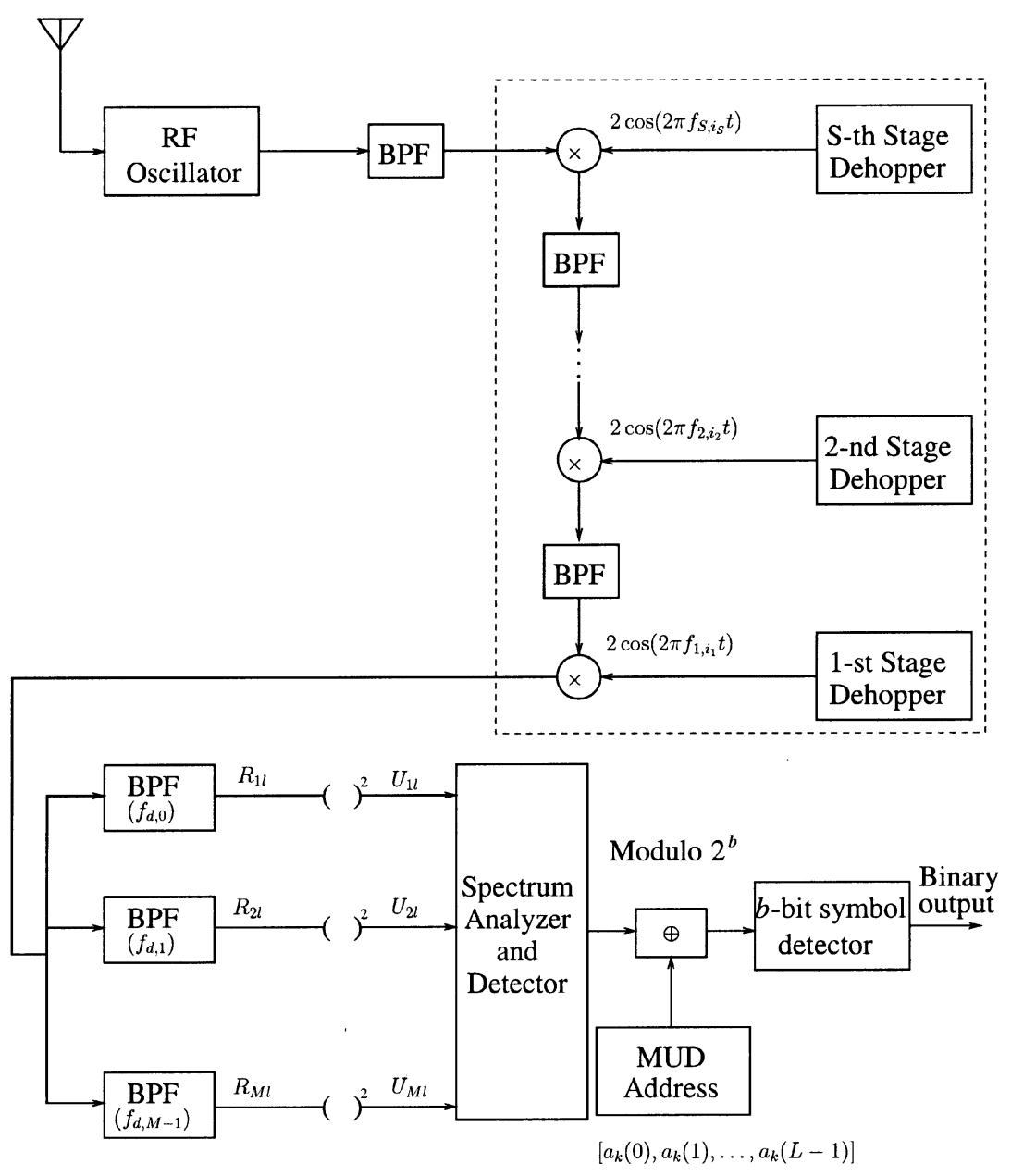

Fig. 3. Receiver block diagram of the MS/UWB FH SSMA system using MFSK modulation.

tones is activated. The hopping rate at each of the $S$ FH stages is $R_{h} / S$. Explicitly, the $\mathrm{FH}$ rate at a given $\mathrm{FH}$ stage is substantially lower, than the $\mathrm{FH}$ rate $R_{h}$ of the overall scheme. The achievable diversity order of the MS/UWB FHMA receiver can reach $L=\omega S$, as argued previously. In our example of Table I, each MFSK symbol is transmitted with the aid of three chips, which are associated with three different composite frequencies. Hence, when encountering frequency-selective fading across the FH bands, a diversity order of three can be achieved by the three-stage FH/UWB FHMA system of Table I using $\omega=1$.

Above, we have discussed the transmitter, the spectrum assignment, the FH strategy, as well as the achievable diversity order of the proposed MS/UWB FHMA system. Let us now consider the detection of the MS/UWB FHMA signals.

\section{DETECTION OF MS/UWB FHMA SIGNALS}

\section{A. Receiver Description}

The receiver schematic of the MS/UWB FHMA system is shown in Fig. 3, which essentially follows the inverse structure of the transmitter. At the receiver, the received signal is first down converted to the intermediate frequency (IF) corresponding to the $\mathrm{FH}$ pattern used. Then, the down-converted signal is frequency dehopped as indicated by the dashed-line box of Fig. 3. In contrast to the FH scheme of the transmitter in Fig. 1, where FH commences at Stage-1 and continues to the $S$ th stage, at the receiver of Fig. 3 the hopping frequencies are removed in the order commencing with the $S$ th stage and finishing at the first-stage, according to the specific FH pattern of a given user. Following the frequency de-hopping stage, the received signal is input to a bank of $M$ energy detectors matched to the $M$ frequency tones of the MFSK stage. The detection interval is $T_{h}$ seconds, which is synchronized with the chips or FH intervals of the desired user. The $M$ energy detectors provide $M L$ outputs during a symbol period of $T_{s}$ seconds. As shown in Fig. 3, the spectrum of each $T_{s}$-duration transmission is analyzed in order to determine, which frequency tone and, hence, which $b$-bit symbol $X_{k}^{(m)}$ was transmitted by invoking the MUD address of the desired user.

\section{B. Conventional Single-User Detection}

The conventional single user detector of fast FHMA using MFSK modulation was investigated by Goodman et al. [18]. In the context of this detector, as described previously, a MUD address is employed at the MFSK stage as a unique signature sequence in order to signal the MFSK tones conveying a $b$-bit symbol of the user considered, as shown in (1). After frequency dehopping and energy detection at the bandpass filter (BPF) 


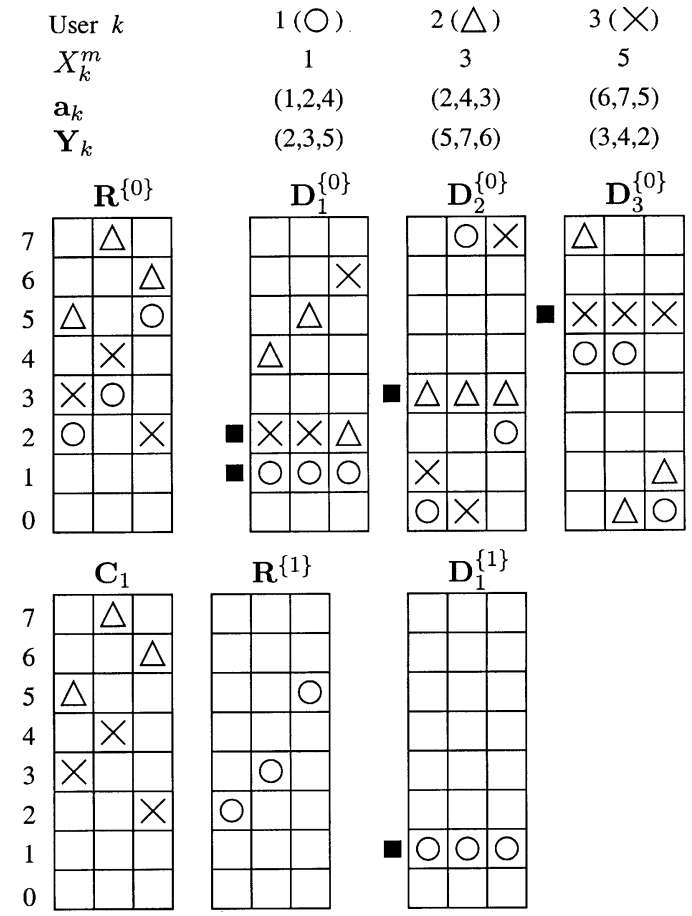

Fig. 4. Single-user (top) and MUD (bottom) example for MS/UWB FHMA, when three users share the same FH address. The eight rows indicate the 8 -ary FSK tones, while the three columns correspond to the three FH intervals or chips of a symbol duration. For example, for $\mathbf{Y}_{1}=(2,3,5)$ and $\mathbf{a}_{1}=(1,2,4)$ using $(6)$, we have $\mathbf{X}=(1,1,1)$; for $\mathbf{Y}_{2}=(5,7,6)$ and $\mathbf{a}_{1}=(1,2,4)$, we have $\mathbf{X}=(4,5,2)$; for $\mathbf{Y}_{3}=(3,4,2)$ and $\mathbf{a}_{1}=(1,2,4)$, we have $\mathbf{X}=(2,2,-2=6)$, etc. Observe that $\mathbf{D}_{2}^{0}$ and $\mathbf{D}_{3}^{0}$ have a unique complete row, yielding a unanimous symbol decision of three and five, respectively, while in case of $\mathbf{D}_{1}^{0}$ we have a 0.5 error probability, when employing conventional single-user detection, as seen in the top row. The MUD technique shown at the bottom eliminates this ambiguity by subtracting the interference matrix $\mathbf{C}_{1}$-created with the aid of the reliable symbol decisions-from $\mathbf{R}_{0}$, yielding the unambiguous matrix $\mathbf{D}_{1}$.

outputs of Fig. 3 with respect to the $L$ chips, the signaling sequence $\mathbf{Y}_{k}$ of (1) is recovered by the receiver. By performing modulo- $\left(M=2^{b}\right)$ subtraction of the unique MUD address $\mathbf{a}_{k}$ of the $k$ th user (desired user) from $\mathbf{Y}_{k}$ on a chip-by-chip basis, we arrive at

$$
X_{k}^{(m)} \cdot \mathbf{1}=\mathbf{Y}_{k} \ominus \mathbf{a}_{k}, \quad k=1,2,3, \ldots
$$

which allows us to recover the transmitted symbol $X_{k}^{(m)}$ of user $k$.

The detection operations associated with the MUD address of the MS/UWB FHMA system can be illustrated by means of time-frequency matrices having $M$ rows and $L$ columns, where the $M$ rows correspond to the $M$ distinct frequency values, while the $L$ columns represent the $L$ number of chips corresponding to one MFSK symbol duration. We refer to the elements of the time-frequency matrices as time-frequency elements. In Fig. $4, \mathbf{R}^{\{0\}}$ represents the received time-frequency matrix of a MS/UWB FHMA system using 8-ary FSK, where we assume that three users share the same FH address (not unique). Provided that neither noise nor fading is present, all elements in the received time-frequency matrix are received correctly. This multiuser channel may also be viewed as a logical OR-channel having legitimate outputs of zero or one for each time-frequency element. Specifically, if at least one chip corresponding to a particular time-frequency element is present at the receiver, the corresponding time-frequency element output is flagged by a logical 1 , otherwise by a logical 0 . The multiuser signal received in a particular time-frequency element is the logical OR function of the corresponding transmitted time-frequency elements of all users sharing the same FH address.

As seen in the matrix $\mathbf{R}^{\{0\}}$ of Fig. 4 representing the received time-frequency matrix of the three users, the signals marked as circles, triangles and crosses are present at the time-frequency elements corresponding to the time-frequency elements activated by users $1-3$, respectively, using 8FSK modulation. Specifically, the tones $(2,3,5),(5,7,6)$, and $(3,4$, 2 ) were activated, which convey the 8 FSK symbols 1,3 , and 5 , respectively, derived below. As an example, we assign the unique MUD signature addresses $(1,2,4),(2,4,3)$, and $(6$, $7,5)$ to users 1,2 , and 3 . The three time-frequency matrices obtained by modulo- 8 subtraction of the unique addresses $\mathbf{a}_{k}$ from $\mathbf{Y}_{k}$ according to (6) for users $k=1,2$, and 3 are denoted by $\mathbf{D}_{1}^{\{0\}}, \mathbf{D}_{2}^{\{0\}}$ as well as $\mathbf{D}_{3}^{\{0\}}$ in Fig. 4. Let us for example employ the majority logic decision (MLD) based decoder of [18], which opts for deciding in favor of the particular 8-ary symbol corresponding to the specific row having the highest number of nonzero entries, for providing an estimate of the transmitted symbol of the users $k=1,2$, and 3 . For example, for users $k=1,2$, or 3 , based on the time-frequency matrices of $\mathbf{D}_{1}^{\{0\}}, \mathbf{D}_{2}^{\{0\}}$ as well as $\mathbf{D}_{3}^{\{0\}}$ in Fig. 4, we find that the transmitted symbol of user 2 was symbol 3 and that of user 3 was symbol 5, which can be correctly decoded with the aid of unambiguous MLD. However, since there are two complete rows in $\mathbf{D}_{1}^{\{0\}}$ and the receiver does not have the explicit knowledge as to which user activated the individual tones, user 1 has to randomly select either symbol 1 or 2 , which results in a symbol error probability of $1 / 2$. Hence, even in the absence of noise, the conventional SUD, which decides concerning the received symbol of each user by ignoring the knowledge of other users, may make erroneous decisions concerning the transmitted symbol, due to the presence of MUI. Therefore, a more potent multiuser detector is required, in order to improve the detection performance of the proposed MS/UWB FHMA system, which is the topic of the next section.

\section{MUD}

It is well-known that a powerful solution of mitigating the effects of the interference caused by nonorthogonal received signals, while also reducing the near-far problem is the employment of MUD [28], where the desired user's symbols are detected by exploiting information concerning the other users. Recently, a wide range of research results has been reported on the subject of MUD in the context of DS-CDMA systems [28]. Typically, the MUD algorithms developed for DS-CDMA systems remove the effects of MUI by exploiting the a priori knowledge concerning the cross correlations between the desired user's signal and each of the interfering signals. A requirement for the operation of these multiuser detectors is the employment of coherent demodulation, which is unfortunately impractical in the context of the fast FHMA system considered. 
Hence, a range of MUD algorithms based on noncoherent demodulation has been proposed for fast FHMA systems. In [29], Timor has presented a MUD algorithm referred to as "stage 2 decoding," which does not require the explicit knowledge of the particular MUD addresses of the active users. Timor also proposed an algorithm referred to as "stage 3 decoding," which exploits the explicit knowledge of the MUD addresses of the active users. By extending the concept of interference cancellation (IC) often employed in DS-CDMA systems [28], in [30] Mabuchi et al. have proposed an innovative MUD scheme for MFSK modulated fast FHMA systems based on the cancellation of the co-channel interference. This MUD scheme can be applied to any set of MUD address codes and it was shown to consistently outperform the conventional single-user receiver. However, when the number of active users is high, this MUD algorithm becomes computationally intensive. In [19], Fiebig has proposed an iterative IC-based MUD algorithm for fast FHMA systems. It was argued that this algorithm reduces the complexity of the solution presented in [30]. In the MUD algorithm proposed by Fiebig [19], the receiver requires the knowledge of all users' MUD addresses, as well as further side-information as to which users are active. Finally, in [20], a multistage MUD algorithm modeled after the multistage detector designed for DS-CDMA systems has been proposed, which removes the MUI by exploiting the knowledge of both the users' MUD addresses and their powers. The multistage MUD algorithm proposed in [20] can be used by both synchronous and asynchronous fast FHMA systems and it was argued that this multiuser detector has a linearly increasing complexity as a function of the number of active users.

The operation of the multiuser detector associated with fast FHMA systems may be understood by referring to the bottom part of Fig. 4. In the previous section, we illustrated that when a conventional SUD is considered, the first user's symbol of 1 might be erroneously detected as symbol 2 , since both row one and row two have three entries. However, based on the MUD algorithms developed in [19] and [20], the MUI inflicted upon the first user's signal by user 2 and 3 can be canceled by jointly exploiting the information of all three users. Specifically, since both the decoded time-frequency matrices $\mathbf{D}_{2}^{\{0\}}$ and $\mathbf{D}_{3}^{\{0\}}$ of Fig. 4 have only one row with three entries, the corresponding symbol 3 of user 2 and symbol 5 of user 3 can be viewed as reliable decisions. Following a tentative first stage detection concerning all reliably detected users, an interference matrix $\mathbf{C}_{1}$ is constructed by reencoding the reliably detected symbol 3 and 5 using the MUD addresses of users 2 and 3, which are shown in the matrix $\mathbf{C}_{1}$ of Fig. 4. Once the interference matrix $\mathbf{C}_{1}$ was generated, the detector compares it with the received matrix of $\mathbf{R}^{\{0\}}$ and subtracts this interference matrix from the received matrix, yielding the matrix $\mathbf{R}^{\{1\}}$ of Fig. 4. Finally, by decoding $\mathbf{R}^{\{1\}}$ using the MUD address $\mathbf{a}_{1}=(1,2,4)$ of user 1 , the time-frequency matrix of $\mathbf{D}_{1}^{\{1\}}$ is obtained. Accordingly, symbol 1 transmitted by the first user can now be reliably detected.

The difference between the MUD algorithm of [19] and that of [20] is that in [19] the time-frequency matrices were constructed based on the hard decision concerning each timefrequency element, while in [20] the time-frequency elements were constituted by the "soft" energy outputs. Hence, the timefrequency matrices in Fiebig's algorithm [19] contain binary " 0 " and "1," while in the algorithm proposed by Halford and BrandtPearce [20] consist of the continuous-valued "soft" quantities. In Fiebig's algorithm [19], the matrix entries constituted by "1" are set to zero, if their corresponding entries in the interference matrix are one. By contrast, according to the algorithm by Halford and Brandt-Pearce [20], the receiver compares the interference matrix to the received matrix and removes a time-frequency element only, if its entry in the received matrix is the same as the corresponding entry in the interference matrix. Furthermore, the multiuser IC procedure of Fig. 4 can be carried out in several consecutive stages, if there are further time-frequency detection matrices, which contain more than one rows having the maximum number of entries.

In the context of the proposed synchronous MS/UWB FHMA system, the above-mentioned MUD algorithms can be employed for enhancing the detection performance. Furthermore, in synchronous MS/UWB FHMA systems the $K$ number of users supported is divided into $M_{S}$ groups and each group hosts only about $K / M_{S} \leq M$ users. Since there is no MUI between users hosted by different groups due to using different frequency bands, the number of users to be detected by a multiuser detector is only about $K / M_{S} \leq M$, which is significantly lower than the total number of users, $K$, in the system. Therefore, the proposed MS/UWB FHMA communication scheme is capable of significantly decreasing the complexity of the multiuser detectors invoked, compared with conventional fast FHMA schemes [19], [20], as well as with respect to DS-CDMA schemes [28] and in comparison to IR based UWB time-hopping multiple access schemes [8].

\section{Simulation Results}

In this section, the BER performance of the MS/UWB FHMA system is studied by simulation for the above mentioned three detection schemes, namely for the conventional SUD, the MUD proposed by Fiebig [19] (MUD: Fiebig), and the MUD proposed by Halford and Brandt-Pearce (MUD: Halford). In our simulations, two types of MUD address assignment schemes are considered, namely the random and the optimum MUD address assignment. In [19] and [20], MUD performance of fast FHMA systems using random MUD address assignment has been investigated. In the context of the random MUD address assignment scheme, each user's MUD addresses are randomly generated. Hence, the BER performance of the MS/UWB FHMA system using the random MUD address assignment scheme is actually the BER performance averaged over all possible address assignment schemes. In the context of the optimum MUD address assignment scheme, the arrangement proposed by Einarsson [31] was invoked in our simulations. Based on this scheme, the $k$ th user's MUD address can be expressed as

$$
\mathbf{a}_{k}=\left(\gamma_{k}, \gamma_{k} \beta, \gamma_{k} \beta^{2}, \ldots, \gamma_{k} \beta^{L-1}\right)
$$

where $\gamma_{k}$ is an element of $G F(M)$ assigned to user $k$ and $\beta$ is a fixed primitive element of $G F(M)$. In our simulations both $G F(16)$ and $G F(32)$ were considered and the corresponding 


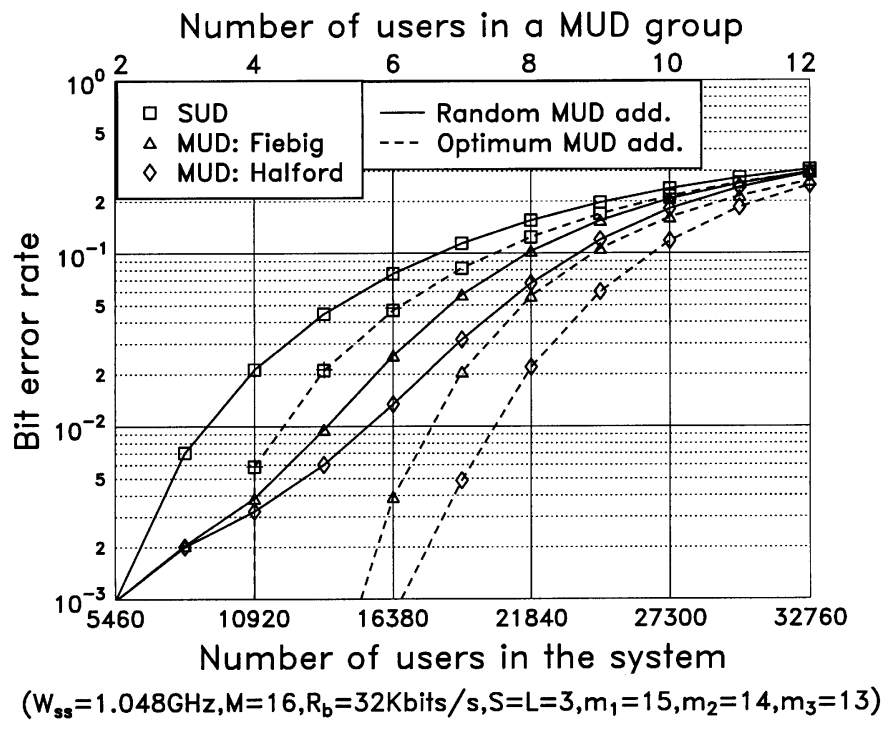

Fig. 5. BER shown for the conventional SUD, for Fiebig's [19] IC multiuser detector (MUD: Fiebig), and for the multiuser detector based on multistage IC [20] (MUD: Halford) in the context of a three-stage UWB FHMA system associated with parameters $m_{1}=15, m_{2}=14, m_{3}=13, M=16$, and $L=S=3$ for transmission over noiseless interference-contaminated channels $\left(E_{b} / N_{0}=\infty\right)$.

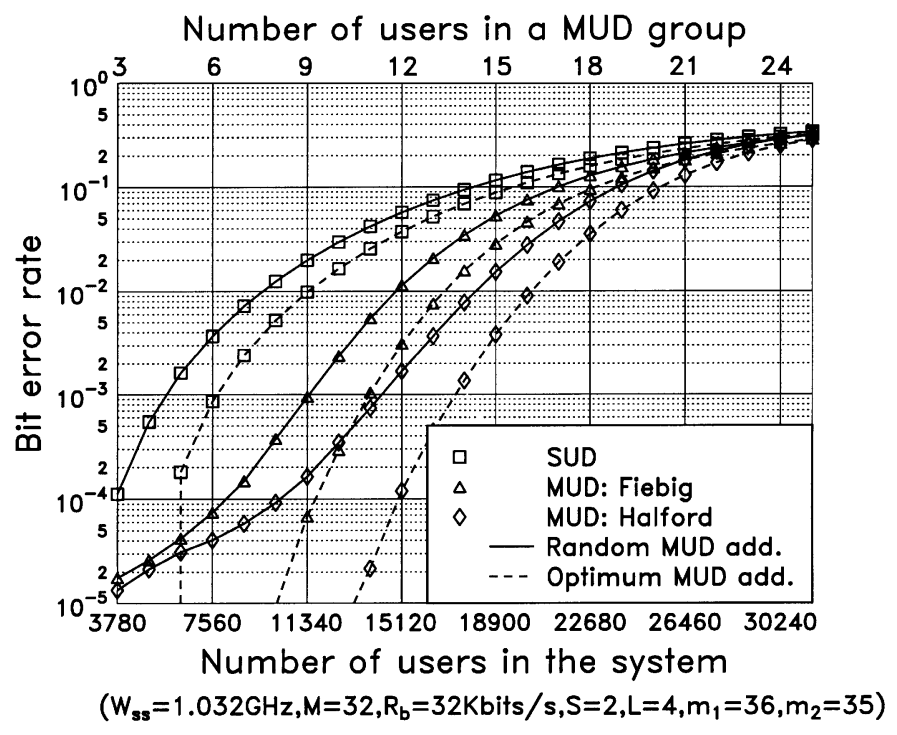

Fig. 6. BER shown for the conventional SUD, for Fiebig's [19] IC multiuser detector (MUD: Fiebig) and for the multiuser detector based on multistage IC [20] (MUD: Halford) in the context of a two-stage UWB FHMA system associated with parameters $m_{1}=36, m_{2}=35, M=32, S=2$, and $L=4$ for transmission over noiseless interference-contaminated channels $\left(E_{b} / N_{0}=\infty\right)$.

primitive polynomials were $p(x)=1+x+x^{4}$ and $p(x)=$ $1+x^{2}+x^{5}$, respectively.

Figs. 5-7 present the BER comparison of the conventional SUD with that of the multiuser detectors using both iterative IC [19] and multistage IC [20] following the first-stage IC, when there is no additive channel noise $\left(E_{b} / N_{0}=\infty\right)$. The BER performance of the above mentioned detection schemes using both the random and the optimum MUD address assignment is also compared. The parameters used in our simulations were listed in the related figures. As shown in Figs. 5-7, we assumed

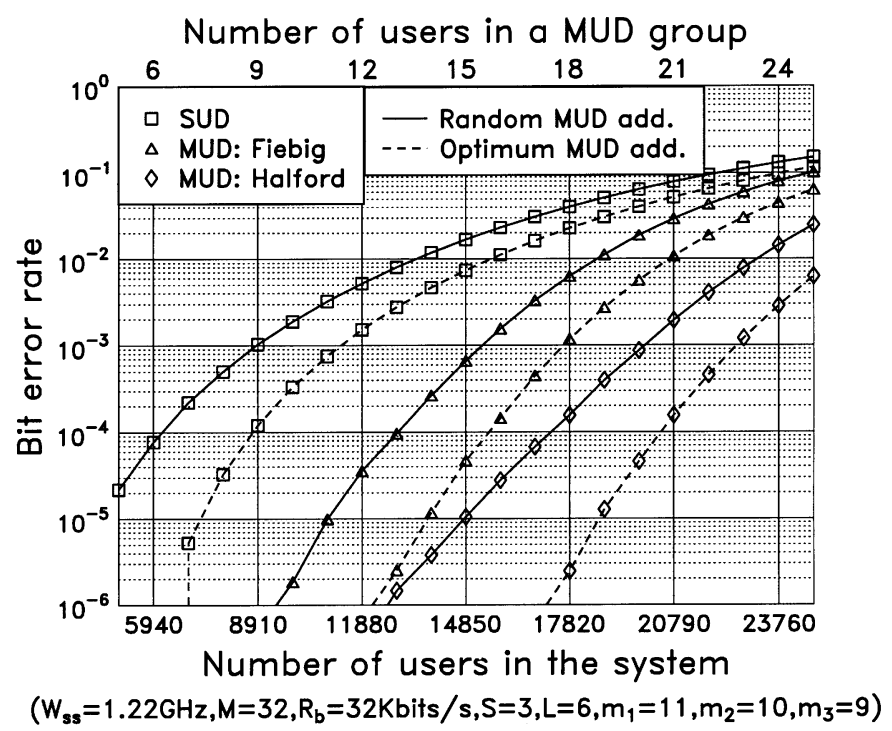

Fig. 7. BER shown for the conventional SUD, for Fiebig's [19] IC multiuser detector (MUD: Fiebig) and for the multiuser detector based on multistage IC [20] (MUD: Halford) in the context of a three-stage UWB FHMA system associated with parameters $m_{1}=11, m_{2}=10, m_{3}=9, M=32, S=3$, and $L=6$ for transmission over noiseless interference-contaminated channels $\left(E_{b} / N_{0}=\infty\right)$.

that the bit rate of each user was $32 \mathrm{~Kb} / \mathrm{s}$ and the total system bandwidth was $1.048 \mathrm{GHz}$ in Fig. 5, $1.032 \mathrm{GHz}$ in Fig. 6, and $1.22 \mathrm{GHz}$ in Fig. 7. The results show that, after the first-stage IC, the multiuser detectors using both iterative IC [19] and multistage IC [20] outperform the conventional SUD by a wide margin. The optimum MUD address assignment significantly outperformed the random MUD address assignment in the context of all three detection schemes. Taking Fig. 5 as an example and considering the target BER of $10^{-2}$, which can be rendered near error free by channel coding, the number of users supported by the MS/UWB FHMA system using iterative IC based MUD [19] associated with both random and optimum MUD addresses is about twice the number supported by the corresponding system using the conventional SUD. Similarly, the number of users supported by the MS/UWB FHMA system using multistage IC based MUD [20] associated with both random and optimum MUD addresses is more than twice the number supported by the corresponding system using the conventional SUD. Furthermore, for the noiseless but interference-contaminated channel considered, the MUD employing multistage IC [20] outperforms iterative IC [19]. Similar results can also be observed from Figs. 6 and 7 . In order that the different MS/UWB FHMA systems using different symbol dynamic ranges of $M=2^{b}$ and different number of chips, $L$, become comparable, in Table II, we summarized the bandwidth efficiency expressed in terms of bits per second per Hertz $(\mathrm{b} / \mathrm{s} / \mathrm{Hz})$. It can be shown that for a given detection scheme, as well as for a given MUD address assignment scheme, the bandwidth efficiency can be optimized by finding the optimum values of $L$ and $M$. For example, for the conventional SUD and for the random MUD address assignment scheme, the bandwidth efficiency of the scheme using $L=4$ and $M=32=2^{5}$ is higher, than that of the schemes using both $L=3, M=16$ and $L=6, M=32$. 
TABLE II

COMPARISON OF BANDWIDTH EFFICIENCY IN TERMS OF THE NUMBER OF BITS PER SECOND PER HERTZ AT THE BER OF $10^{-3}$ FOR THE SYSTEMS CONSIDERED IN Figs. 5-7 USING THE CONVENTIONAL SingLE-USER DETECTOR, AND THE MULTIUSER DETECTORS PROPOSED IN [19] AS WELL AS IN [20]

\begin{tabular}{c|c|c|c|c}
\hline & & \multicolumn{3}{|c}{ Bandwidth efficiency (bits/s/Hz) } \\
\hline \multirow{4}{*}{$\begin{array}{c}\text { Number of } \\
\text { chips per } \\
\text { symbol }\end{array}$} & $\begin{array}{c}\text { Conventional } \\
\text { single-user } \\
\text { detector }\end{array}$ & $\begin{array}{c}\text { Multiuser } \\
\text { detector } \\
\text { I [19] }\end{array}$ & $\begin{array}{c}\text { Multiuser } \\
\text { detector } \\
\text { II [20] }\end{array}$ \\
\hline \multirow{2}{*}{$\begin{array}{c}\text { Mundom } \\
\text { addresses }\end{array}$} & $L=3(M=16)$ & 0.17 & 0.17 & 0.17 \\
\cline { 2 - 5 } & $L=4(M=32)$ & 0.20 & 0.35 & 0.44 \\
\cline { 2 - 5 } $\begin{array}{c}\text { Optimum } \\
\text { MUD }\end{array}$ & $L=6(M=32)$ & 0.18 & 0.40 & 0.53 \\
\cline { 2 - 5 } addresses & $L=4(M=16)$ & 0.33 & 0.46 & 0.51 \\
\cline { 2 - 5 } & $L=6(M=32)$ & 0.30 & 0.46 & 0.54 \\
\hline
\end{tabular}

However, for the multiuser detector employing multistage IC [20] and optimum address assignment, the scheme using $L=6$ and $M=32$ achieves the highest bandwidth efficiency. Note that although the total number of users supported by the MS/UWB FHMA system may be as high as tens of thousands, the number of users to be detected by each multiuser detector is extremely low, as shown at the top of Figs. 5-7. Therefore, the employment of powerful multiuser detectors is affordable for the proposed MS/UWB FHMA system, in order to attain a near-single-user performance.

In Fig. 5, it can be observed that when the number of users in a group is two and when random MUD address assignment is invoked, the conventional SUD and the multiuser detectors using both iterative IC and multistage IC reach the same error probability. This is not a coincidence, since it can be explained as follows. For the conventional SUD employing the random MUD address assignment scheme for supporting two users and in the absence of channel impairment, the only event resulting an erroneous symbol decision is, when there exist two complete rows in the time-frequency detection matrix $\mathbf{D}_{i}, i=1,2$ of the desired user. Careful consideration of this scenario reveals that this ambiguous arises, when the two randomly generated MUD addresses, namely $\mathbf{a}_{1}=\left[a_{1}(0), a_{1}(1), \ldots, a_{1}(L-1)\right]$ of user 1 and $\mathbf{a}_{2}=\left[a_{2}(0), a_{2}(1), \ldots, a_{2}(L-1)\right]$ of user 2 obey the relationship of $a_{1}(0)-a_{2}(0)=a_{1}(1)-a_{2}(1)=\cdots=$ $a_{1}(L-1)-a_{2}(L-1)=C \neq 0$. Due to the above relationship, it can be shown that if there exist two complete rows in the time-frequency detection matrix $\mathbf{D}_{1}$ for user 1 , there exist two complete rows also in the time-frequency detection matrix $\mathbf{D}_{2}$ of user 2. Hence, no symbols can be reliably detected using the conventional SUD. Since no reliable symbols can be provided for supporting IC, multiuser IC cannot be carried out. Therefore, the conventional SUD and the iterative IC, and multistage IC based MUD achieve the same error probability. Furthermore, when the number of users is higher than two, the MUD addresses obeying the relationship of $a_{1}(0)-a_{j}(0)=a_{i}(1)-$ $a_{j}(1)=\cdots=a_{i}(L-1)-a_{j}(L-1)=C \neq 0$ also degrade the BER performance of the IC based MUDs. Hence, in a practical fast FHMA system the MUD addresses having the above property must be avoided. Since the MUD addresses based on the optimum assignment scheme [31] obey the relationship of $a_{i}(0)-a_{j}(0) \neq a_{i}(1)-a_{j}(1) \neq \cdots \neq a_{i}(L-1)-a_{j}(L-1)$ for any $i \neq j$, as shown in the Figs. 5-7, in the absence of channel impairment, the BER equals zero, when the number of users is not higher than the number of chips, i.e., when $k \leq L$.

\section{CONCLUSION AND Future RESEARCH}

In summary, we have proposed a novel multiple-access scheme, namely MS/UWB FHMA, which is suitable for ultra-wideband wireless communications using FFH techniques in conjunction with MFSK modulated narrowband signals. The spectrum assignment, the system design parameters, the FH strategy, and the appropriate detection schemes as well as the achievable BER performance have been investigated, when communicating over MUI contaminated channels. Then, a RNS assisted synchronous FFH scheme has been proposed, which is capable of efficiently dividing the huge number of users supported by the synchronous MS/UWB FHMA system into a number of groups, where each group contains only a small fraction of the total number of users supported. Hence, MUI is only experienced by the users within each group. Hence, the receiver of the synchronous MS/UWB FHMA system can afford invoking advanced MUD algorithms, in order to increase its capacity. By contrast, without employing user groups the MUD complexity would be excessive. It has been shown that the MS/UWB FHMA system is capable of supporting a tremendous number of users, while employing low-complexity receivers.

The philosophy of MS/UWB FHMA can be readily extended to a range of other multiple-access schemes associated with different modulation arrangements. Specifically, the first-stage FH subbands shown in Fig. 2, which host the MFSK tones can be configured to support different modulation as well as spreading schemes. For example, the first-stage FH bands can be used for DS spreading, which results in a multiple-access scheme that may be referred to as MS/UWB FH/DS CDMA, which essentially belongs to the family of UWB hybrid DS/FH CDMA schemes. Similarly, when the MS/UWB FH/DS CDMA scheme is designed for using slow FH, where several symbols are transmitted during one FH interval, DS spreading associated with a wide range of modulation schemes, such as binary phase-shift keying (BPSK), quarternay phase-shift keying (QPSK), $M$-ary orthogonal modulation, etc., can be employed. However, when the MS/UWB FH/DS CDMA scheme is designed for using fast FH - as in this paper-in order to exploit the advantages of fast FH, DS spreading associated with noncoherent modulation schemes has to be employed due to the associated complexity of phase tracking imposed by fast FHMA systems. In MS/UWB FHMA, the first-stage FH bands, as shown in Fig. 2, can also be occupied by various multicarrier CDMA signals [32]. These types of UWB multiple-access schemes can be referred to as MS/UWB FH/MC CDMA. Similarly to the MS/UWB FH/DS CDMA scheme, appropriate data modulation schemes and multicarrier modulation schemes have to be selected, in order to meet various system design requirements in the context of slow FH or fast FH. The design and performance characterization of these systems constitutes the subject of our future work. 


\section{REFERENCES}

[1] D. Bertsekas and R. Gallager, Data Networks, 2nd ed. Englewood Cliffs, NJ: Prentice-Hall, 1992.

[2] M. Zeng, A. Annamalai, and V. K. Bhargava, "Recent advances in cellular wireless communications," IEEE Commun. Mag., vol. 27, pp. 128-138, Sept. 1999.

[3] M. Progler, C. Evci, and M. Umehira, "Air interface access schemes for broadband mobile systems," IEEE Commun. Mag., vol. 37, pp. 106-115, Sept. 1999.

[4] R. A. Scholtz and M. Z. Win, "Impulse radio," in Wireless Communication-TDMA Versus CDMA, S. G. Glisic and P. L. Leppanen, Eds. Norwell, MA: Kluwer, 1997, pp. 245-267.

[5] M. Z. Win and R. A. Scholtz, "On the energy capture of ultrawide bandwidth signals in dense multipath environments," IEEE Commun. Lett., vol. 2, pp. 245-247, Sept. 1998

[6] — - "Impulse radio: How it works," IEEE Commun. Lett., vol. 2, pp. 36-38, Feb. 1998.

[7] M. Z. Win, X. Qiu, R. A. Scholtz, and O. K. Li, "ATM-based TH-SSMA network for multimedia PCS," IEEE J. Select. Areas Commun., vol. 17, pp. 824-836, May 1999.

[8] M. Z. Win and R. A. Scholtz, "Ultra-wide bandwidth time-hopping spread-spectrum impulse radio for wireless multiple-access communications," IEEE Trans. Commun., vol. 48, pp. 679-691, Apr. 2000.

[9] M. Sablatash, "Advances in ultra-wideband communications: Description, performance, and advantages," in Proc. Wireless, Calgary, AB, Canada, July 8-10, 2002, pp. 228-247.

[10] M. Sushchik et al., "Chaotic pulse position modulation: A robust method of communicating with chaos," IEEE Commun. Lett., vol. 4, pp. $128-130$, Apr. 2000.

[11] M. G. M. Hussain, "Principles of space-time array processing for ultrawide-band impulse radar and radio communications," IEEE Trans. Veh. Technol., vol. 51, pp. 393-403, May 2002.

[12] O. Wintzell, D. K. Zigangirov, and K. S. Zigangirov, "On the capacity of a pulse-position-hopped CDMA system," IEEE Trans. Inform. Theory, vol. 47, pp. 2639-2644, Sept. 2001

[13] F. Ramirez-Mireles, "On the performance of ultra-wide-band signals in Gaussian noise and dense multipath," IEEE Trans. Veh. Technol., vol. 50, pp. 244-249, Jan. 2001.

[14] M. A. Soderstrand, W. K. Jenkins, and G. A. Jullien, Residue Number System Arithmetic: Modern Applications in Digital Signal Processing. Piscataway, NJ: IEEE Press, 1986.

[15] L.-L. Yang and L. Hanzo, "Performance of residue number system based DS-CDMA over multipath fading channels using orthogonal sequences," Eur. Trans. Telecommun., vol. 9, pp. 525-536, Nov.-Dec. 1998.

[16] - "Residue number system arithmetic assisted $M$-ary modulation," IEEE Commun. Lett., vol. 3, pp. 28-30, Feb. 1999.

[17] — "Performance analysis of coded $M$-ary orthogonal signaling using errors-and-erasures decoding over frequency-selective fading channels," IEEE J. Select. Areas Commun., vol. 19, pp. 211-221, Feb. 2001.

[18] D. J. Goodman, P. S. Henry, and V. K. Prabhu, "Frequency-hopped multilevel FSK for mobile radio," Bell Syst. Tech. J., vol. 59, pp. 1257-1275, Sept. 1980.

[19] U. C. G. Fiebig, "Iterative interference cancellation for FFH/MFSK MA systems," in Proc. IEE Communications, vol. 143, Dec. 1996, pp. 380-388.

[20] K. W. Halford and M. Brandt-Pearce, "Multistage multiuser detection for FHMA," IEEE Trans. Commun., vol. 48, pp. 1550-1562, Sept. 2000.

[21] T. Vlachus and E. Geraniotis, "Performance study of hybrid spread-spectrum random-access communications," IEEE Trans. Commun., vol. 39, pp. 975-985, June 1991.

[22] M. K. Simon, J. K. Omura, R. A. Scholtz, and B. K. Levitt, SpreadSpectrum Communications. Rockville, MD: Comput. Sci., 1985, vol. I.

[23] P. V. Kumar, "Frequency-hopping code sequence designs having large linear span," IEEE Trans. Inform. Theory, vol. 34, pp. 146-151, Jan. 1988.

[24] S. V. Marie and E. L. Titlebaum, "A class of frequency hop codes with nearly ideal characteristics for use in multiple-access spread-spectrum communications and radar and sonar systems," IEEE Trans. Commun., vol. 40, pp. 1442-1447, Sept. 1992.
[25] L. Bin, "One-coincidence sequences with specified distance between adjacent symbols for frequency-hopping multiple access," IEEE Trans. Commun., vol. 45, pp. 408-410, Apr. 1997.

[26] R. W. Watson and C. W. Hastings, "Self-checked computation using residue arithmetic," Proc. IEEE, vol. 54, pp. 1920-1931, Dec. 1966.

[27] H. Krishna and J.-D. Sun, "On theory and fast algorithms for error correction in residue number system product codes," IEEE Trans. Comput., vol. 42, pp. 840-852, July 1993.

[28] S. Verdu, Multiuser Detection. Cambridge, U.K.: Cambridge Univ Press, 1998

[29] U. Timor, "Improved decoding scheme for frequency-hopped multilevel FSK system," Bell Syst. Tech. J., vol. 59, pp. 1839-1855, 1980.

[30] T. Mabuchi, R. Kohno, and H. Imai, "Multiuser detection scheme based on canceling cochannel interference for MFSK/FHSSMA system," IEEE J. Select. Areas Commun., vol. 12, pp. 593-604, May 1994.

[31] G. Einarsson, "Address assignment for a time-frequency-coded, spreadspectrum system," Bell Syst. Tech. J., vol. 59, pp. 1241-1255, Sept. 1980.

[32] L.-L. Yang and L. Hanzo, "Performance of generalized multicarrier DS-CDMA over Nakagami-m fading channels," IEEE Trans. Commun., vol. 50, pp. 956-966, June 2002.

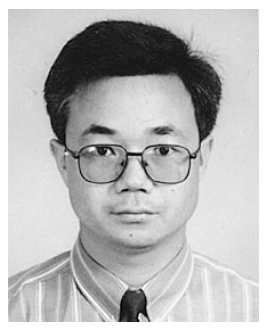

Lie-Liang Yang (M'98-SM'02) received the B.Eng. degree in communication engineering from Shanghai TieDao University, Shanghai, China, in 1988, and the M.S and Ph.D. degrees in communications and electronics from Northern Jiaotong University, Beijing, China, in 1991 and 1997, respectively.

From June 1997 to December 1997, he was a Visiting Scientist at the Institute of Radio Engineering and Electronics, Academy of Sciences of the Czech Republic. Since December 1997, he has been with the Communication Group, Department of Electronics and Computer Science, University of Southampton, Southampton, U.K. and has been involved in researching various error-correction coding, modulation, and detection techniques, as well as wideband, broadband, and ultra-wideband CDMA systems for the advanced wireless mobile communication systems. He has published over 70 papers in journals and conference proceedings.

Dr. Yang was awarded the Royal Society Sino-British Fellowship in 1997.

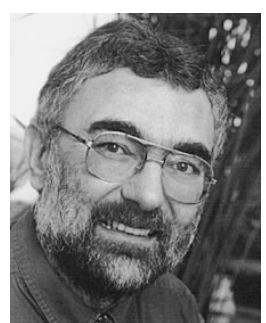

Lajos Hanzo (M'91-SM'92) received the Master's degree in electronics in 1976 and the doctorate in 1983 from the Technical University of Budapest, Hungary.

During his 26-year career in telecommunications, he has held various research and academic posts in Hungary, Germany, and the U.K. Since 1986, he has been with the Department of Electronics and Computer Science, University of Southampton, Southampton, U.K., where he holds the Chair in telecommunications. He has coauthored eight books on mobile-radio communications, published about 400 research papers, organized and chaired conference sessions, presented overview lectures. Currently, he is managing an academic research team, working on a range of research projects in the field of wireless multimedia communications sponsored by industry, the Engineering and Physical Sciences Research Council (EPSRC) U.K., the European IST Programme, and the Mobile Virtual Center of Excellence (VCE), U.K. He is an enthusiastic supporter of industrial and academic liaison and he offers a range of industrial courses. For further information on research in progress and associated publications please refer to http://www-mobile.ecs.soton.ac.uk

Dr. Hanzo is an IEEE Distinguished Lecturer and has been awarded a number of distinctions. 